\title{
Objective Response by mRECIST Is an Independent Prognostic Factor for Overall Survival in Hepatocellular Carcinoma Treated with Sorafenib in the SILIUS Trial
}

\author{
Masatoshi Kudo ${ }^{a} K_{\text {Kazuomi Ueshima }}{ }^{a}$ Yasutaka Chiba $^{a}$ Sadahisa Ogasawara ${ }^{b}$ \\ Shuntaro Obic Namiki Izumi ${ }^{d}$ Hiroshi Aikatae Hiroaki Nagano ${ }^{f}$ \\ Etsuro Hatano ${ }^{g}$ Yutaka Sasaki $^{\text {h }}$ Keisuke Hino ${ }^{i}$ Takashi Kumadaj \\ Kazuhide Yamamotok ${ }^{k}$ Yasuharu Imail Shouta Iwadou ${ }^{m}$ Chikara Ogawa $^{n}$ \\ Takuji Okusaka $^{\circ}$ Fumihiko Kanai $^{\text {b }}$ Yasuaki Arai $^{\circ}$ \\ ${ }^{a}$ Kindai University Faculty of Medicine, Osaka-Sayama, Japan; ${ }^{b}$ Chiba University Graduate \\ School of Medicine, Chiba, Japan; 'Kyoundo Hospital, Tokyo, Japan; dJapanese Red Cross \\ Musashino Hospital, Musashino, Japan; e Hiroshima University Hospital, Hiroshima, Japan; \\ ${ }^{f}$ Osaka University Graduate School of Medicine, Osaka, Japan; ${ }^{9}$ Kyoto University, Graduate \\ School of Medicine, Kyoto, Japan; h'Kumamoto University Graduate School of Medical \\ Sciences, Kumamoto, Japan; i Kawasaki Medical School, Kurashiki, Japan; jOgaki Municipal \\ Hospital, Ogaki, Japan; ' Okayama University Medical School, Okayama, Japan; 'Ikeda \\ Municipal Hospital, Ikeda, Japan; ${ }^{m}$ Hiroshima City Hospital, Hiroshima, Japan; ${ }^{\text {n Takamatsu }}$ \\ Red Cross Hospital, Takamatsu, Japan; ${ }^{\circ}$ National Cancer Center Hospital, Tokyo, Japan
}

\section{Keywords}

Hepatocellular carcinoma · Objective response · Modified RECIST · Sorafenib · Hepatic arterial infusion chemotherapy

\begin{abstract}
Objective: In SILIUS (NCT01214343), combination of sorafenib and hepatic arterial infusion chemotherapy did not significantly improve overall survival (OS) in patients with advanced hepatocellular carcinoma (HCC) compared with sorafenib alone. In this study, we explored the relationship between objective response by MRECIST and OS in the sorafenib group, in the combination group, and in all patients in the SILIUS trial. Methods: Association between objective response and OS in patients treated with sorafenib $(n=103)$ or combination $(n=102)$ and all patients $(n=205)$ were analyzed. The median OS of responders was compared with that of non-responders. Landmark analyses were performed according to objective response at several fixed time points, as sensitivity analyses, and the effect on OS was evaluated by Cox regression analysis with objective response as a time-dependent covariate, with other prog-
\end{abstract}


Kudo et al.: Objective Response by Systemic Therapy Is an Independent Prognostic Factor for OS

nostic factors. Results: In the sorafenib group, OS of responders $(n=18)$ was significantly better than that of non-responders $(n=78)(p<0.0001)$, where median OS was $27.2(95 \% \mathrm{Cl}$, 16.0-not reached) months for responders and $8.9(95 \% \mathrm{Cl}, 6.5-12.6)$ months for non-responders. HRs from landmark analyses at 4,6 , and 8 months were $0.45(p=0.0330), 0.37(p=0.0053)$, and $0.36(p=0.0083)$, respectively. Objective response was an independent predictor of OS based on unstratified Cox regression analyses. In the all patients and the combination group, similar results were obtained. Conclusions: In the SILIUS trial, objective response by sorafenib assessed by mRECIST is an independent prognostic factor for OS in patients with HCC.

(C) 2019 S. Karger AG, Base

\section{Introduction}

Hepatocellular carcinoma (HCC) is the third leading cause of cancer deaths globally [1, 2]. In Japan, more than $60 \%$ of HCC cases are detected early enough to be eligible for hepatectomy or ablation [3]. However, globally, most cases are diagnosed as advanced HCCs that are no longer resectable [4, 5]. Currently, sorafenib and lenvatinib [6] are the first-line systemic agents approved worldwide for the treatment of advanced HCC.

Sorafenib is a multi-kinase inhibitor for which survival benefit was proven in the phase III SHARP trial [7] and the phase III Asia Pacific trial [8]. Lenvatinib is also a multi-kinase inhibitor, with a particularly strong inhibitory effect on VEGFR1-3, FGFR1-4, PDGFR $\alpha$, RET, and KIT, and blocks angiogenesis and tumor growth [9-12].

The two major regimens used for hepatic arterial infusion chemotherapy (HAIC) are low-dose cisplatin plus 5-fluorouracil (5FU), and 5FU plus a systemic interferon, both of which are widely used in Japan, South Korea, and Taiwan [13]. Several retrospective comparative cohort studies have demonstrated a survival benefit of HAIC over no treatment in advanced HCC patients with vascular invasion or multiple liver lesions [14-20]. Furthermore, the Liver Cancer Study Group of Japan conducted a propensity score-matched analysis of data from a nationwide follow-up study and demonstrated a survival benefit of HAIC over best supportive care in 476 HCC patients [21]. In addition, phase I/II prospective studies also suggested the favorable results of HAIC in advanced HCC $[22,23]$.

However, HAIC has not yet been tested in a prospective randomized phase III clinical trial, and thus it is not globally regarded as standard of care. Meanwhile, Kudo et al. [24] conducted the prospective, controlled phase III SILIUS trial, which compared overall survival (OS) between sorafenib alone and sorafenib plus HAIC (5FU and cisplatin). The authors found that addition of HAIC to sorafenib did not significantly improve OS (the primary endpoint) in patients with advanced HCC. However, subgroup analysis revealed a better objective response rate (ORR) in the sorafenib plus HAIC group (36\% in intention-to-treat [ITT] cohort) than in the sorafenib alone group (18\% in the ITT cohort) in ITT analysis. Also, time to progression was significantly better in the sorafenib plus HAIC group than in the sorafenib alone group, demonstrating that sorafenib plus HAIC had a stronger antitumor effect than that of sorafenib alone. Furthermore, stratification by portal vein invasion (Vp0, Vp1-3, or Vp4, denoting no, first-to-third branch, or main portal vein invasion, respectively) revealed that sorafenib plus HAIC tended to result in a greater survival benefit compared with sorafenib alone specifically in Vp4 patients [24].

In the SILIUS trial, modified Response Evaluation Criteria in Solid Tumors (mRECIST) was used to assess ORR, and the ORR to sorafenib plus HAIC was significantly better than the ORR to sorafenib alone. It is well known that objective response (OR) to transarterial chemoembolization (TACE), ablation, or molecular targeted therapy does not correlate with OS if OR is assessed using the standard RECIST1.1. To address this issue, Lencioni et al. $[25,26]$ 


\section{Liver Cancer}

\begin{tabular}{|c|c|}
\hline \multicolumn{2}{|l|}{ Liver Cancer 2019;8:505-519 } \\
\hline DOI: 10.1159/000503032 & $\begin{array}{l}\text { (c) } 2019 \text { S. Karger AG, Basel } \\
\text { www.karger.com/lic }\end{array}$ \\
\hline
\end{tabular}

Kudo et al.: Objective Response by Systemic Therapy Is an Independent Prognostic Factor for OS

developed the mRECIST to assess the response to treatment for HCC. The mRECIST regards necrotic tissue as an effect of treatment and distinguishes necrotic tumor tissue from residual viable tumor tissue by size measurement on dynamic contrast-enhanced computed tomography or magnetic resonance imaging. A meta-analysis of 7 studies showed that OR to TACE and ablation, as assessed by mRECIST, correlated well with OS [27], and subsequently the European Association for the Study of the Liver (EASL) used this in its guidelines as evidence that OR assessed by mRECIST to locoregional therapy is a prognostic factor for OS [2]. Similarly, OR to molecular targeted therapy, as assessed by mRECIST, was an independent prognostic factor as well as a predictive factor, based on retrospective analyses of 2 previous studies $[26,28]$. Nevertheless, as stated in the EASL guidelines, additional data are needed to confirm the relationship between OR to systemic therapy and OS [2] since from a statistical point of view the usual methods of comparing responders with non-responders were wrong due to guarantee-time bias or immortal time bias, leading to biased estimates of the survival distributions, invalid statistical tests, and misleading conclusions $[29,30]$.

In addition, the Journal of Clinical Oncology decided to no longer publish articles that include survival by tumor response based on simple responder analysis. An editorial accompanying the letter by the editor of the Journal of Clinical Oncology indicated that "authors should not compare survival of responders and non-responders without discussing the limitations of such a comparison" [31]. Therefore, the usual method of responder analysis has been regarded as a highly biased method, and almost all biostatical specialists or oncologists have recommended not to perform it to identify OR as an independent predictor or prognostic factor of cancer treatment. Actually, Simon and Wittes [32] indicated in a guideline that comparisons of survival by tumor response in clinical trials should not be published because of the enormous biases. In 2013, Giobbie-Hurder et al. [33] published the statistical methodology paper that there are several analytical methodologies that can remove the "Guaranteetime bias or immortal bias": (1) landmark analysis at the several fixed time points, (2) Cox regression analysis using $\mathrm{OR}$ as a time-dependent covariate, (3) inverse probability weighing, and (4) use of the Mantel-Byar test instead of the log rank test [34]. In this article, the relationship between OR assessed by mRECIST and OS in sorafenib and sorafenib plus HAIC was examined using the phase 3 clinical trial database of the SILIUS study to determine whether OR evaluated by mRECIST is a predictive and prognostic factor for OS in HCC. As stated earlier, the usual method of responder analysis was not used. Instead, we followed Giobbie-Hurder's statistical methods, which are currently becoming acceptable statistical methods to exclude the potential guarantee-time bias. In this article, we used Mantel-Byar test for overall responder analysis and performed landmark analysis at 3 fixed time points $(4,6$, and 8 months). Also, we performed Cox regression multivariate analysis using OR as a timedependent covariate and used a weighted Kaplan-Meier estimate.

\section{Patients and Methods}

SILIUS Trial Design and Assessments

The SILIUS study [24] is a phase III multicenter, open-label, prospective, randomized controlled trial of patients with unresectable HCC (ClinicalTrials.gov, No. NCT01214343). A total of 205 patients were assigned in a 1:1 ratio to sorafenib alone $(n=102)$ or sorafenib plus HAIC $(n=103)$. The starting dose of sorafenib was $800 \mathrm{mg} /$ day. In the sorafenib plus HAIC group, cisplatin was administered at $20 \mathrm{mg} / \mathrm{m}^{2}$ per day on days 1 and 8 , and fluorouracil was administered at a dose of $330 \mathrm{mg} / \mathrm{m}^{2}$ per day on days 1-5 and 8-12 of every 28-day cycle, followed by 2 weeks off treatment. The first treatment cycle was started within 28 days of randomization. Sorafenib was continued until patients progressed, as assessed by mRECIST, or their general health worsened. Key inclusion criteria were as follows: age $\geq 20$ years; advanced HCC untreatable by hepatectomy, local ablation, or TACE; life expectancy $\geq 12$ weeks; Eastern Cooperative Oncology Group (ECOG) performance status of 0 or 
Kudo et al.: Objective Response by Systemic Therapy Is an Independent Prognostic Factor for OS

1; Child-Pugh score $\leq 7$; and adequate bone marrow, liver, and renal function. The key exclusion criterion was the presence of another previous or current malignancy. Stratification factors were institution, the presence or absence of an extrahepatic metastasis, and macroscopic vascular invasion (Vp0, Vp1-3, or Vp4). All patients provided written informed consent. The study protocol, amendments of the protocol, and the informed consent were approved by the ethics committees or Institutional Review Boards of individual participating institutions. This clinical trial was conducted in compliance with the Declaration of Helsinki and the Good Clinical Practice Guidelines. The primary endpoint was OS, defined as the length of time from randomization to death from any cause. Secondary endpoints were progression-free survival, time to progression, ORR, and safety. Computed tomography was performed every 8 weeks to assess the treatment effect.

Overall, 96 of 103 patients in the sorafenib group (93.3\%) and 82 of 102 patients in the sorafenib plus HAIC group (80.4\%) were evaluable for response with a baseline and at least one on-study scan. In this study, unlike in the original report [24], patients in whom OR was not evaluable were excluded from subanalysis.

\section{Statistical Analysis}

Analyses were performed using the SAS 9.4 software package.

This post-hoc retrospective study compared investigator-assessed OR by mRECIST to OS in the total study population, in the sorafenib alone group, and in the sorafenib plus HAIC group. Responders were defined as those who had a CR or PR, and non-responders as those who had stable disease (SD) or progressive disease (PD). The difference of investigator-assessed OR between the two groups was evaluated by Fisher's exact test. OS in responders and non-responders in the sorafenib alone group and in the sorafenib plus HAIC group was estimated by the Kaplan-Meier method. In the total patient population, the OS was estimated by the weighted Kaplan-Meier method with treatment (sorafenib alone or sorafenib plus HAIC) as the weight. In the sorafenib alone and the sorafenib plus HAIC groups, the unweighted Kaplan-Meier method was used. The Mantel-Byar test was used to assess statistical significance. Landmark analysis of OS in each group by OR status was conducted at 4, 6, and 8 months after the initiation of therapy. The log-rank test was used for the inference associated to the landmark analysis. HRs and the 95\% CIs were calculated from Cox models with all variables assessed as time-fixed covariates except OR, which was analyzed as a time-dependent covariate. Significant factors identified by univariable analysis were tested in multivariable analyses. A multivariable Cox regression model with OR as a time-dependent covariate was used to explore prognostic factors of OS.

\section{Results}

\section{Efficacy}

At the end of follow-up, 165 of the 205 patients had died, with a median OS of 11.5 months (95\% CI, 8.2-14.8) in patients treated with sorafenib alone and 11.8 months (95\% CI, 9.1-14.5) in those treated with sorafenib plus HAIC (HR, 1.009 [95\% CI, 0.743-1.371]; $p=0.955)$. In the total patient population, $30.9 \%$ of patients $(55 / 178)$ were responders, and the remaining $69.1 \%$ (123/178) were non-responders. In the sorafenib alone group, $18.8 \%$ of patients (18/96) were responders, and the remaining 81.2\% (78/96) were non-responders. In the sorafenib plus HAIC group, $45.1 \%$ of patients (37/82) were responders and the remaining $54.9 \%$ (45/82) were non-responders. Baseline and clinical characteristics of responders and non-responders are shown in Table 1.

\section{OR as an Independent Prognostic Factor}

Total SILIUS Patient Population

In all patients, the median OS was 25.7 months (95\% CI, 17.3-33.4) in responders and 9.3 months (95\% CI, 6.9-11.4) in non-responders. The HR was 0.31 (95\% CI, 0.20-0.46; $p<$ 0.0001). ORR assessed by mRECIST was 30.9\% (55/178; 95\% CI, 24.2-38.3) in the SILIUS population. The ORR was significantly higher in the sorafenib plus HAIC group than in the sorafenib alone group; investigator-assessed ORR by mRECIST was 45.1\% (95\% CI, 34.156.5 ) in the sorafenib plus HAIC group and 18.8\% (95\% CI, 11.5-28.0) in the sorafenib alone 


\section{Liver Cancer}

\begin{tabular}{l|l}
\hline \multicolumn{2}{l}{ Liver Cancer 2019;8:505-519 } \\
\hline DOI: 10.1159/000503032 & $\begin{array}{l}\text { (c) 2019 S. Karger AG, Basel } \\
\text { www.karger.com/lic }\end{array}$ \\
\hline
\end{tabular}

Kudo et al.: Objective Response by Systemic Therapy Is an Independent Prognostic Factor for OS

Table 1. Patient characteristics of responders and non-responders in the SILIUS trial

\begin{tabular}{|c|c|c|c|c|c|c|}
\hline \multirow[t]{2}{*}{ Characteristics } & \multicolumn{2}{|l|}{ All cohort } & \multicolumn{2}{|c|}{ Sorafenib alone group } & \multicolumn{2}{|c|}{ Sorafenib plus HAIC group } \\
\hline & $\begin{array}{l}\text { responders } \\
(n=55)\end{array}$ & $\begin{array}{l}\text { non-responders } \\
(n=123)\end{array}$ & $\begin{array}{l}\text { responders } \\
(n=18)\end{array}$ & $\begin{array}{l}\text { non-responders } \\
(n=78)\end{array}$ & $\begin{array}{l}\text { responders } \\
(n=37)\end{array}$ & $\begin{array}{l}\text { non-responders } \\
(n=45)\end{array}$ \\
\hline \multicolumn{7}{|l|}{ Age, $n(\%)$} \\
\hline$\geq 65$ years & $35(64)$ & $82(67)$ & $14(78)$ & $54(69)$ & $21(57)$ & $28(62)$ \\
\hline$<65$ years & $20(36)$ & $41(33)$ & $4(22)$ & $24(31)$ & $16(43)$ & $17(38)$ \\
\hline \multicolumn{7}{|l|}{ Sex, $n(\%)$} \\
\hline Male & $47(85)$ & $108(88)$ & $15(83)$ & $67(86)$ & $32(86)$ & $41(91)$ \\
\hline Female & $8(15)$ & $15(12)$ & 3 (17) & $11(14)$ & $5(14)$ & $4(9)$ \\
\hline \multicolumn{7}{|l|}{ Performance status, $n(\%)$} \\
\hline 0 & $50(91)$ & 107 (87) & $17(94)$ & $69(88)$ & $33(89)$ & $38(84)$ \\
\hline 1 & $5(9)$ & $16(13)$ & $1(6)$ & $9(12)$ & $4(11)$ & $7(16)$ \\
\hline \multicolumn{7}{|l|}{ Etiology HBV, $n(\%)$} \\
\hline Yes & $14(25)$ & $28(23)$ & $4(22)$ & 15 (19) & $10(27)$ & $13(29)$ \\
\hline No & $40(73)$ & $94(76)$ & $13(72)$ & $62(79)$ & $27(73)$ & $32(71)$ \\
\hline Indeterminate & $1(2)$ & $1(1)$ & $1(6)$ & $1(1)$ & $0(0)$ & $0(0)$ \\
\hline \multicolumn{7}{|l|}{ Etiology HCV, $n(\%)$} \\
\hline Yes & $26(47)$ & $54(44)$ & $9(50)$ & $34(44)$ & $17(46)$ & $20(44)$ \\
\hline No & $28(51)$ & $67(54)$ & $8(44)$ & $43(55)$ & $20(54)$ & $24(53)$ \\
\hline Indeterminate & $1(2)$ & $2(2)$ & $1(6)$ & $1(1)$ & $0(0)$ & $1(2)$ \\
\hline \multicolumn{7}{|l|}{ ALBI grade, $n(\%)$} \\
\hline 1 & $24(44)$ & $40(33)$ & $9(50)$ & $28(36)$ & $15(41)$ & $12(27)$ \\
\hline 2 & $31(56)$ & $82(67)$ & $9(50)$ & $50(64)$ & 22 (59) & $32(71)$ \\
\hline 3 & $0(0)$ & $1(1)$ & $0(0)$ & $0(0)$ & $0(0)$ & $1(2)$ \\
\hline \multicolumn{7}{|l|}{ Albumin, $n(\%)$} \\
\hline$\geq 3.7 \mathrm{~g} / \mathrm{dL}$ & $34(62)$ & $62(50)$ & $10(56)$ & $43(55)$ & $24(65)$ & $19(42)$ \\
\hline$<3.7 \mathrm{~g} / \mathrm{dL}$ & $21(38)$ & $61(50)$ & $8(44)$ & $35(45)$ & $13(35)$ & $26(58)$ \\
\hline \multicolumn{7}{|l|}{ Bilirubin, $n(\%)$} \\
\hline$<0.8 \mathrm{mg} / \mathrm{dL}$ & $33(60)$ & $53(43)$ & $15(83)$ & $30(38)$ & $18(49)$ & $23(51)$ \\
\hline$\geq 0.8 \mathrm{mg} / \mathrm{dL}$ & $22(40)$ & $70(57)$ & $3(17)$ & $48(62)$ & $19(51)$ & $22(49)$ \\
\hline \multicolumn{7}{|l|}{$\begin{array}{l}\text { Macroscopic portal } \\
\quad \text { vein invasion, } n(\%)\end{array}$} \\
\hline Vp0 & $23(42)$ & $49(40)$ & 7 (39) & $30(38)$ & $16(43)$ & $19(42)$ \\
\hline Vp1-4 & $32(58)$ & $74(60)$ & $11(61)$ & $48(62)$ & 21 (57) & $26(58)$ \\
\hline \multicolumn{7}{|c|}{ Extrahepatic spread, $n(\%)$} \\
\hline Yes & $15(27)$ & $31(25)$ & $5(28)$ & $19(24)$ & $10(27)$ & $12(27)$ \\
\hline No & $40(73)$ & $92(75)$ & $13(72)$ & $59(76)$ & $27(73)$ & $33(73)$ \\
\hline \multicolumn{7}{|c|}{ Baseline serum AFP, $n(\%)$} \\
\hline$<400 \mathrm{ng} / \mathrm{mL}$ & $30(55)$ & $63(51)$ & $11(61)$ & $41(53)$ & $19(51)$ & $22(49)$ \\
\hline$\geq 400 \mathrm{ng} / \mathrm{mL}$ & $23(42)$ & $60(49)$ & $7(39)$ & 37 (47) & $16(43)$ & $23(51)$ \\
\hline Missing & $2(4)$ & $0(0)$ & $0(0)$ & $0(0)$ & $2(5)$ & $0(0)$ \\
\hline \multicolumn{7}{|c|}{ Baseline serum DCP, $n(\%)$} \\
\hline$<2,050 \mathrm{mAU} / \mathrm{mL}$ & 27 (49) & $61(50)$ & $9(50)$ & $42(54)$ & $18(49)$ & $19(42)$ \\
\hline$>2,050 \mathrm{mAU} / \mathrm{mL}$ & $25(45)$ & $62(50)$ & $8(44)$ & $36(46)$ & $17(46)$ & $26(58)$ \\
\hline Missing & $3(5)$ & $0(0)$ & $1(6)$ & $0(0)$ & $2(5)$ & $0(0)$ \\
\hline \multicolumn{7}{|l|}{ mRECIST BOR, $n(\%)$} \\
\hline Complete response & $10(18)$ & $0(0)$ & $2(11)$ & $0(0)$ & $8(22)$ & $0(0)$ \\
\hline Partial response & $45(82)$ & $0(0)$ & $16(89)$ & $0(0)$ & $29(78)$ & $0(0)$ \\
\hline Stable disease & $0(0)$ & $86(70)$ & $0(0)$ & $57(73)$ & $0(0)$ & $29(64)$ \\
\hline Progressive disease & $0(0)$ & $37(30)$ & $0(0)$ & 21 (27) & $0(0)$ & $16(36)$ \\
\hline
\end{tabular}


Kudo et al.: Objective Response by Systemic Therapy Is an Independent Prognostic Factor for OS

Table 1 (continued)

\begin{tabular}{|c|c|c|c|c|c|c|}
\hline \multirow[t]{2}{*}{ Characteristics } & \multicolumn{2}{|l|}{ All cohort } & \multicolumn{2}{|c|}{ Sorafenib alone group } & \multicolumn{2}{|c|}{ Sorafenib plus HAIC group } \\
\hline & $\begin{array}{l}\text { responders } \\
(n=55)\end{array}$ & $\begin{array}{l}\text { non-responders } \\
(n=123)\end{array}$ & $\begin{array}{l}\text { responders } \\
(n=18)\end{array}$ & $\begin{array}{l}\text { non-responders } \\
(n=78)\end{array}$ & $\begin{array}{l}\text { responders } \\
(n=37)\end{array}$ & $\begin{array}{l}\text { non-responders } \\
(n=45)\end{array}$ \\
\hline \multicolumn{7}{|l|}{ Treatment, $n(\%)$} \\
\hline Sorafenib + HAIC & $37(67)$ & $45(37)$ & & & & \\
\hline Sorafenib & $18(33)$ & $78(63)$ & & & & \\
\hline
\end{tabular}

ALBI grade, albumin-bilirubin grade; AFP, alfa-fetoprotein; DCP, des- $\gamma$-carboxy prothrombin; BOR, best objective response; HAIC, hepatic arterial infusion chemotherapy.

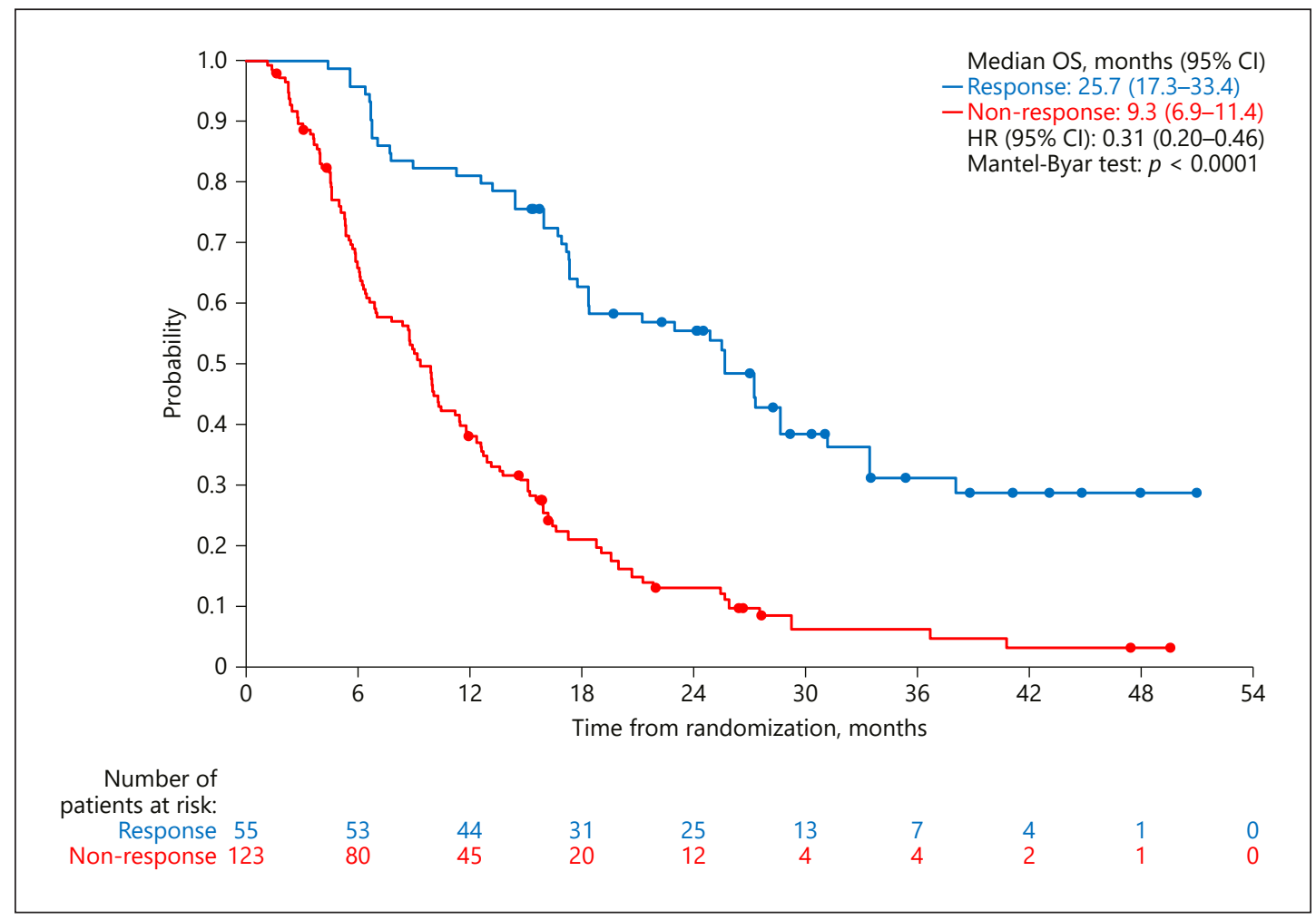

Fig. 1. OS by OR assessed by mRECIST for the overall SILIUS population. Mantel-Byar test was used to exclude the Guarantee-time bias.

group ( $p=0.0001$, Fisher's exact test). Kaplan-Meier estimates in responders and in nonresponders in the overall SILIUS trial patients are shown in Figure 1.

Landmark analyses at 4,6, and 8 months showed an overall OS benefit in responders compared with non-responders. Landmark analysis at 4 months after randomization revealed that OS was significantly longer in patients with OR than in those without OR (HR, 0.54 [95\% CI, 0.34-0.87]; $p=0.0106$ ). The same was true at 6 months (HR, 0.41 [95\% CI, 0.26-0.66]; $p=0.0003$ ) and 8 months (HR, 0.36 [95\% CI, 0.22-0.58]; $p<0.0001$ ) (Fig. 2).

Multivariable Cox regression analysis revealed that OR assessed by mRECIST was an independent prognostic factor (HR, 0.37 [95\% CI, 0.24-0.57]; $p<0.0001$ ). Other prognostic factors identified were ECOG PS (HR, 1.88 [95\% CI, 1.15-3.08]; $p=0.0122$ ) and alpha fetoprotein (AFP) level (HR, 1.84 [95\% CI, 1.31-2.60]; $p=0.0005$ ] (Table 2). 
Kudo et al.: Objective Response by Systemic Therapy Is an Independent Prognostic Factor for OS

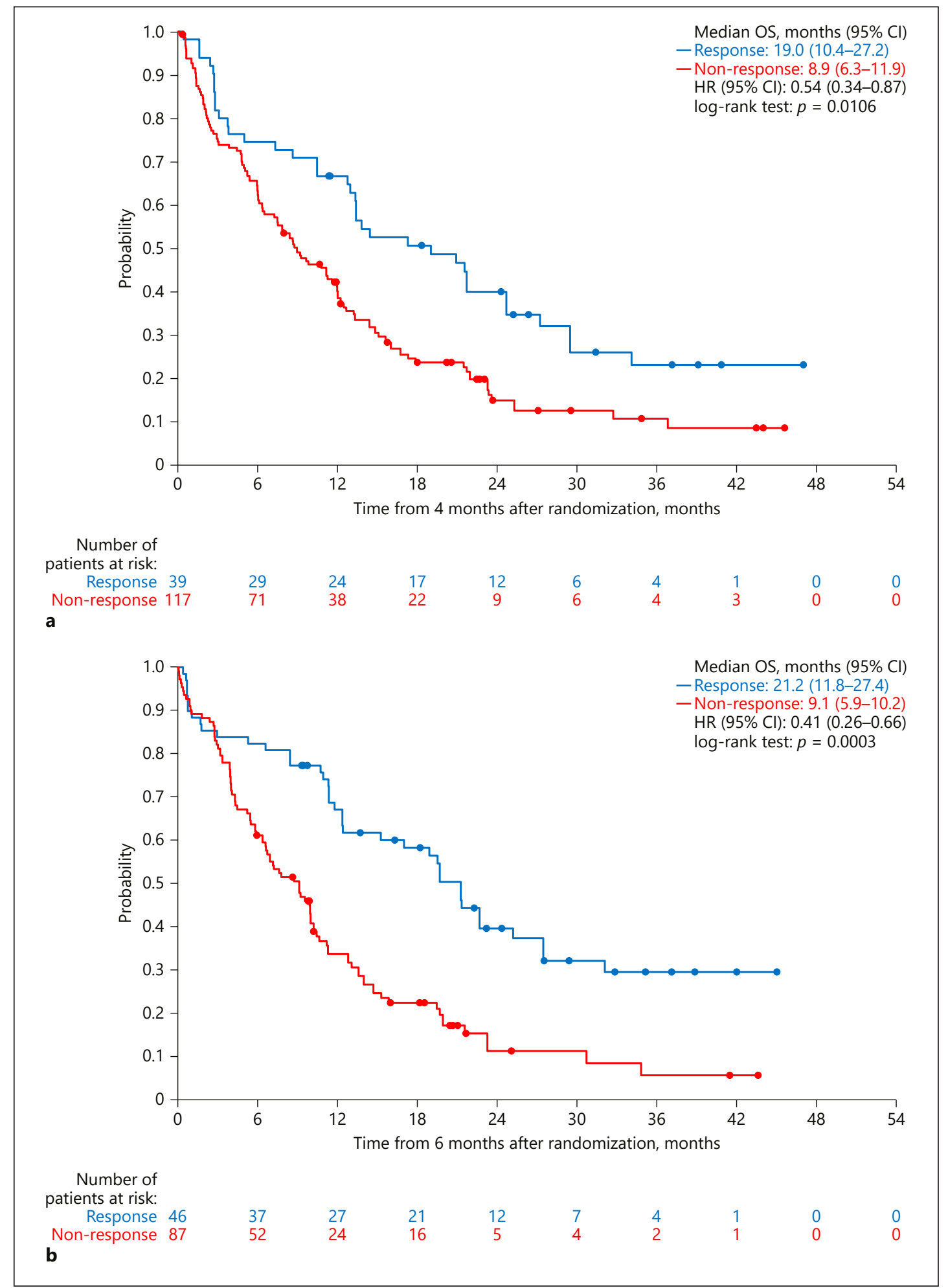

Fig. 2. a Landmark analyses for OS by OR assessed by mRECIST in the overall SILIUS population (landmark Kaplan-Meier curve as function of tumor response at 4 months). b Landmark analyses for OS by OR assessed by mRECIST in the overall SILIUS population (landmark Kaplan-Meier curve as function of tumor response at 6 months). c Landmark analyses for OS by OR assessed by mRECIST in the overall SILIUS population (landmark Kaplan-Meier curve as function of tumor response at 8 months). NR, not reached.

(Figure continued on next page.) 
Kudo et al.: Objective Response by Systemic Therapy Is an Independent Prognostic Factor for OS

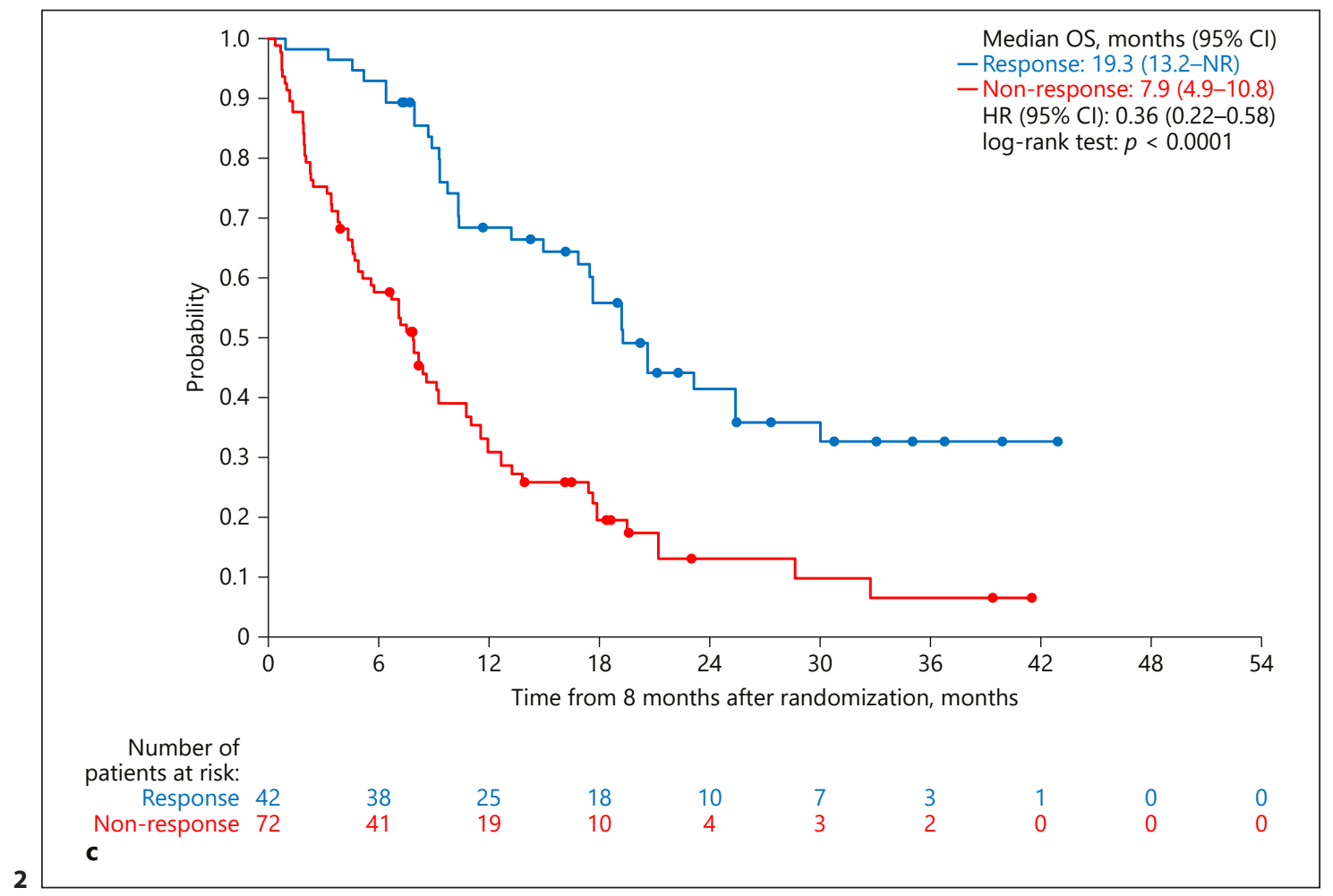

Table 2. Univariable and multivariable analysis of factors associated with OS (overall SILIUS)

\begin{tabular}{|c|c|c|c|c|}
\hline \multirow[t]{2}{*}{ Parameter } & \multicolumn{2}{|c|}{ Univariable analysis } & \multicolumn{2}{|c|}{ Multivariable analysis } \\
\hline & HR (95\% CI) & $p$ value & $\operatorname{HR}(95 \% \mathrm{CI})$ & $p$ value \\
\hline Treatment (SOR+HAIC vs. SOR) & $0.88(0.63-1.23)$ & 0.4521 & & \\
\hline Response (CR+PR vs. SD+PD) & $0.39(0.26-0.58)$ & $<0.0001$ & $0.37(0.24-0.57)$ & $<0.0001$ \\
\hline Age $(\geq 65$ vs. $<65)$ & $1.25(0.87-1.78)$ & 0.2245 & & \\
\hline Sex (male vs. female) & $0.76(0.47-1.22)$ & 0.2575 & & \\
\hline PS ( 1 vs. 0$)$ & $2.17(1.34-3.51)$ & 0.0015 & $1.88(1.15-3.08)$ & 0.0122 \\
\hline Vp (Vp1-4 vs. Vp0) & $0.88(0.63-1.22)$ & 0.4384 & & \\
\hline Extrahepatic spread (yes vs. no) & $1.03(0.70-1.51)$ & 0.8981 & & \\
\hline HBV (yes vs. no) & $1.15(0.78-1.71)$ & 0.4753 & & \\
\hline HCV (yes vs. no) & $0.93(0.66-1.30)$ & 0.6741 & & \\
\hline Albumin $(\geq 3.6$ vs. $<3.6 \mathrm{mg} / \mathrm{dL})$ & $0.80(0.58-1.12)$ & 0.1976 & & \\
\hline Bilirubin ( $\geq 0.8$ vs. $<0.8 \mathrm{mg} / \mathrm{dL}$ ) & $1.57(1.12-2.21)$ & 0.0086 & $1.34(0.95-1.89)$ & 0.0995 \\
\hline ALBI grade (grade 2 vs. grade 1 ) & $1.26(0.88-1.79)$ & 0.2019 & & \\
\hline $\operatorname{AFP}(\geq 400$ vs. $<400 \mathrm{ng} / \mathrm{mL})$ & $1.68(1.20-2.36)$ & 0.0024 & $1.84(1.31-2.60)$ & 0.0005 \\
\hline DCP $(\geq 2,050$ vs. $<2,050 \mathrm{mAU} / \mathrm{mL})$ & $1.05(0.75-1.47)$ & 0.7610 & & \\
\hline
\end{tabular}

All covariates were time-fixed except for response, which was time-dependent. OS, overall survival; ALBI grade, albumin-bilirubin grade; AFP, alfa-fetoprotein; DCP, des- $\gamma$-carboxy prothrombin.

Sorafenib Alone Group

The median OS was 27.2 months (95\% CI, 16.0-not reached) in responders and 8.9 months $(95 \% \mathrm{CI}, 6.5-12.6)$ in non-responders in the sorafenib alone group. The HR was 0.32 (95\% CI, 0.17-0.62; $p<0.0001)$. ORR assessed by mRECIST was $18.8 \%(18 / 96$; $95 \%$ CI, $11.5-$ 
Kudo et al.: Objective Response by Systemic Therapy Is an Independent Prognostic Factor for OS

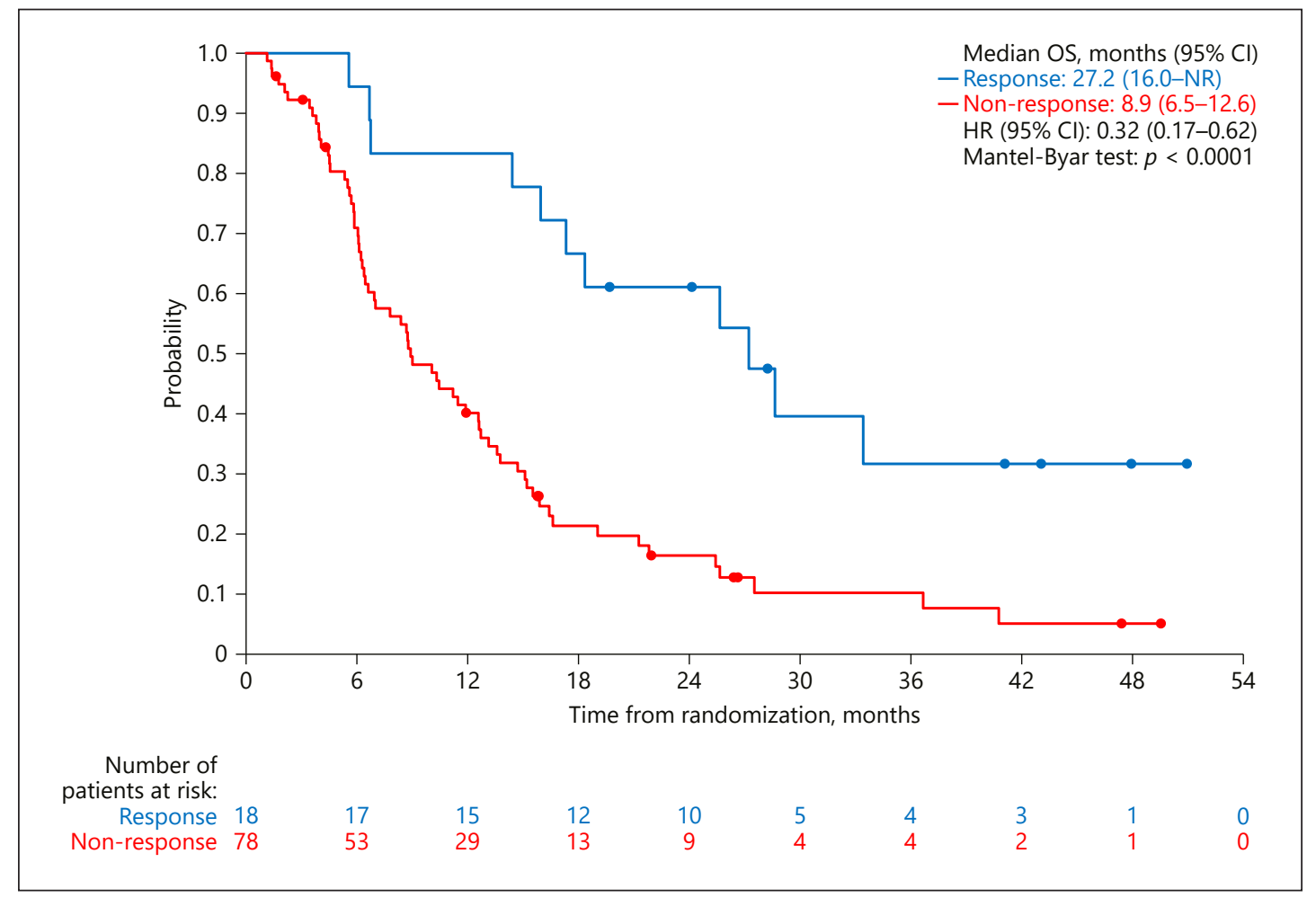

Fig. 3. OS by OR assessed by mRECIST for the sorafenib alone group. Mantel-Byar test was used to exclude the Guarantee-time bias. NR, not reached.

28.0). Kaplan-Meier estimates in responders and in non-responders in sorafenib alone group $\mathrm{l}$ are shown in Figure 3.

Landmark analyses at each time point showed an overall OS benefit in responders compared with non-responders. Landmark analysis at 4 months after randomization revealed that OS was significantly longer in patients with OR than in those without OR (HR, 0.45 [95\% $\mathrm{CI}, 0.21-0.96] ; p=0.0330$ ). This was also the case at 6 months (HR, 0.37 [95\% CI, 0.18-0.76]; $p=0.0053$ ) and at 8 months (HR, 0.36 [95\% CI, 0.17-0.79]; $p=0.0083$ ) (Fig. 4).

Multivariable Cox regression analysis revealed that OR assessed by mRECIST was an independent prognostic factor (HR, 0.38 [95\% CI, 0.18-0.84]; $p=0.0164$ ). AFP level was also identified as an independent prognostic factor (HR, 1.70 [95\% CI, 1.02-2.83]; $p=0.0406$ ) (Table 3).

Sorafenib plus HAIC Group

The median OS was 23.0 months (95\% CI, 16.9-31.2) in responders and 9.9 months ( $95 \%$ CI, 5.3-11.8) in non-responders in the sorafenib plus HAIC group. The HR was 0.28 (95\% CI, $0.16-0.49 ; p<0.0001)$. ORR assessed by mRECIST was $45.1 \%$ (18/96; 95\% CI, 11.5-28.0). Kaplan-Meier estimates in responders and in non-responders in the sorafenib plus HAIC group are shown in online supplementary Figure 1 (for all online suppl. material, see www. karger.com/doi/10.1159/000503032).

Landmark analyses at 4, 6, and 8 months showed an overall OS benefit in responders compared with non-responders. Land mark analyses at 4 months after randomization revealed that OS was not significantly longer in patients with OR than in those without OR (HR, 0.74; $95 \%$ CI, $0.42-1.28 ; p=0.2752$ ). However, the median OS was significantly better in patients 
Kudo et al.: Objective Response by Systemic Therapy Is an Independent Prognostic Factor for OS

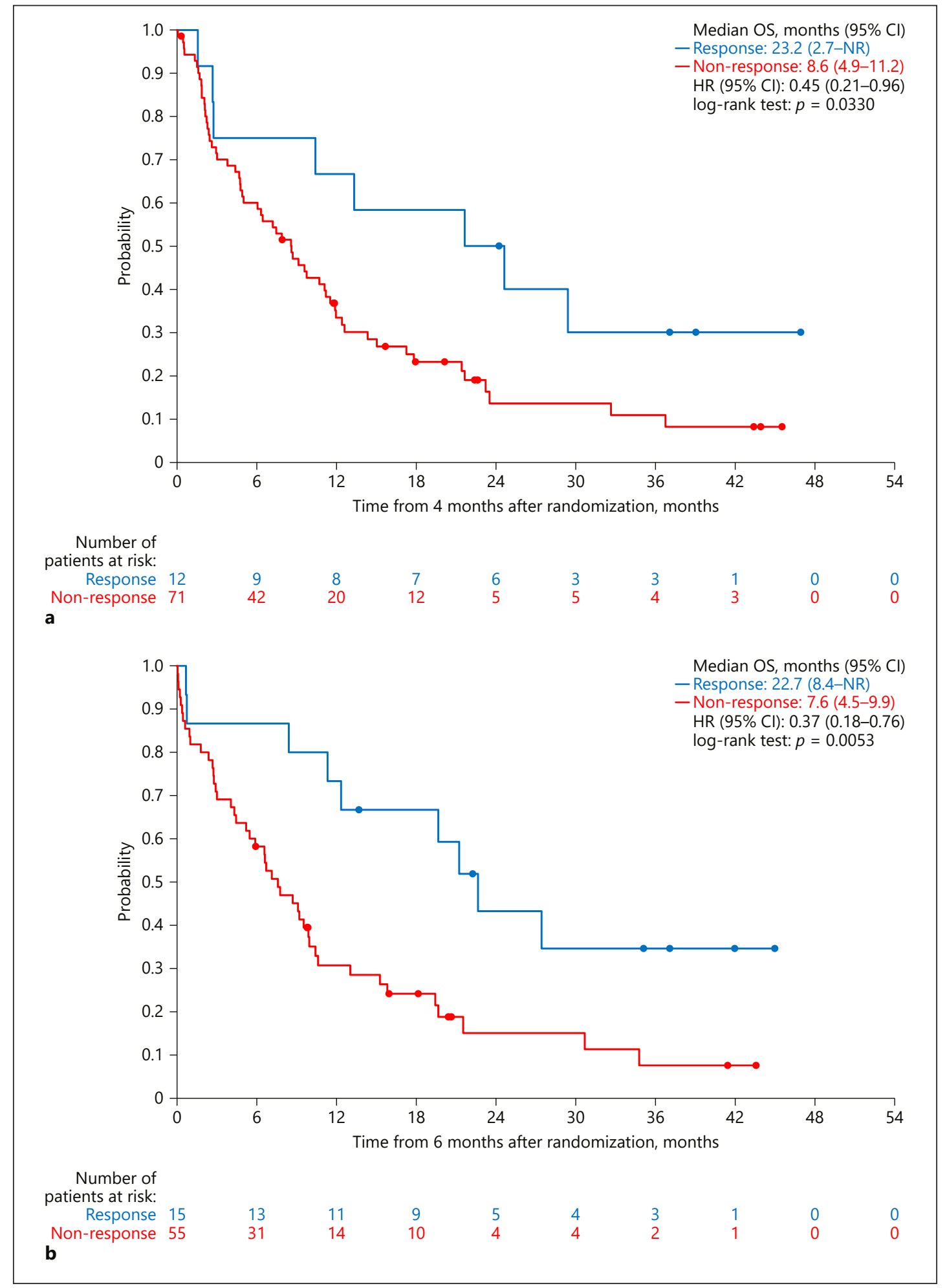

Fig. 4. a Landmark analyses for OS by OR assessed by mRECIST in the sorafenib alone group (landmark Kaplan-Meier curve as function of tumor response at 4 months). b Landmark analyses for OS by OR assessed by mRECIST in the sorafenib alone group (landmark Kaplan-Meier curve as function of tumor response at 6 months). c Landmark analyses for OS by OR assessed by mRECIST in the sorafenib alone group (landmark Kaplan-Meier curve as function of tumor response at 8 months). NR, not reached.

(Figure continued on next page.) 
Kudo et al.: Objective Response by Systemic Therapy Is an Independent Prognostic Factor for OS

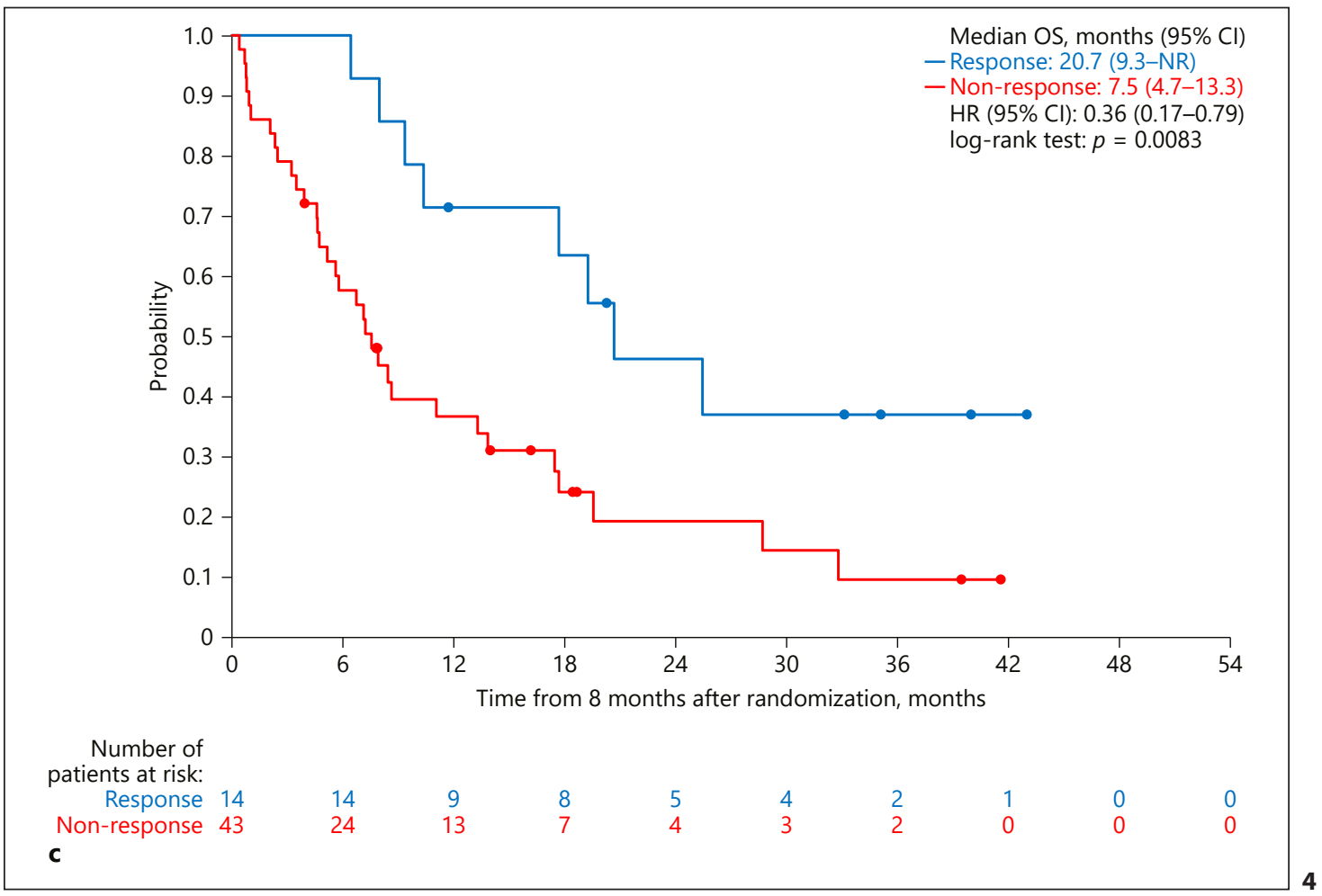

Table 3. Univariable and multivariable analysis of factors associated with OS (sorafenib alone group)

\begin{tabular}{|c|c|c|c|c|}
\hline \multirow[t]{2}{*}{ Parameter } & \multicolumn{2}{|c|}{ Univariable analysis } & \multicolumn{2}{|c|}{ Multivariable analysis } \\
\hline & $\mathrm{HR}(95 \% \mathrm{CI})$ & $p$ value & $\operatorname{HR}(95 \% \mathrm{CI})$ & $p$ value \\
\hline Response (CR+PR vs. SD+PD) & $0.37(0.19-0.71)$ & 0.0029 & $0.38(0.18-0.84)$ & 0.0164 \\
\hline Age ( $\geq 65$ vs. $<65)$ & $0.94(0.57-1.53)$ & 0.7936 & & \\
\hline Sex (male vs. female) & $1.06(0.56-2.01)$ & 0.8550 & & \\
\hline PS ( 1 vs. 0$)$ & $1.95(1.00-3.80)$ & 0.0509 & $1.88(0.91-3.88)$ & 0.0880 \\
\hline Vp (Vp1-4 vs. Vp0) & $0.90(0.57-1.42)$ & 0.6502 & & \\
\hline Extrahepatic spread (yes vs. no) & $1.14(0.68-1.92)$ & 0.6205 & & \\
\hline HBV (yes vs. no) & $1.77(1.01-3.10)$ & 0.0472 & $1.39(0.71-2.72)$ & 0.3381 \\
\hline HCV (yes vs. no) & $0.68(0.43-1.08)$ & 0.0997 & $0.86(0.50-1.49)$ & 0.5989 \\
\hline Albumin ( $\geq 3.6$ vs. $<3.6 \mathrm{mg} / \mathrm{dL})$ & $1.10(0.70-1.74)$ & 0.6839 & & \\
\hline Bilirubin ( $\geq 0.8$ vs. $<0.8 \mathrm{mg} / \mathrm{dL}$ ) & $1.94(1.21-3.09)$ & 0.0055 & $1.35(0.80-2.26)$ & 0.2608 \\
\hline ALBI grade (grade 2 vs. grade 1 ) & $0.92(0.58-1.45)$ & 0.7071 & & \\
\hline $\operatorname{AFP}(\geq 400$ vs. $<400 \mathrm{ng} / \mathrm{mL})$ & $1.48(0.94-2.33)$ & 0.0884 & $1.70(1.02-2.83)$ & 0.0406 \\
\hline DCP $(\geq 2,050$ vs. $<2,050 \mathrm{mAU} / \mathrm{mL})$ & $1.04(0.67-1.64)$ & 0.8500 & & \\
\hline
\end{tabular}

All covariates were time-fixed except for response, which was time-dependent. OS, overall survival; ALBI grade, albumin-bilirubin grade; AFP, alfa-fetoprotein; DCP, des- $\gamma$-carboxy prothrombin.

with OR than in those without OR at 6 months (HR, 0.54; 95\% CI, 0.29-0.99; $p=0.0439$ ) and at 8 months (HR, 0.36; 95\% CI, 0.22-0.58; $p<0.0001$ ) (online suppl. Fig. 2).

Multivariable Cox regression analysis revealed that OR assessed by mRECIST was an independent prognostic factor ( $\mathrm{HR}, 0.32$ [95\% CI, 0.18-0.59]; $p=0.0003$ ). AFP level was also a significant prognostic factor (HR 2.46 [95\% CI, 1.33-4.55], $p=0.0043$ ) (Table 4). 
Kudo et al.: Objective Response by Systemic Therapy Is an Independent Prognostic Factor for OS

Table 4. Univariable and multivariable analysis of factors associated with OS (sorafenib plus HAIC group)

\begin{tabular}{|c|c|c|c|c|}
\hline \multirow[t]{2}{*}{ Parameter } & \multicolumn{2}{|c|}{ Univariable analysis } & \multicolumn{2}{|c|}{ Multivariable analysis } \\
\hline & $\operatorname{HR}(95 \% \mathrm{CI})$ & $p$ value & $\operatorname{HR}(95 \% \mathrm{CI})$ & $p$ value \\
\hline Response (CR+PR vs. SD+PD) & $0.36(0.21-0.64)$ & 0.0004 & $0.32(0.18-0.59)$ & 0.0003 \\
\hline Age ( $\geq 65$ vs. $<65$ ) & $1.63(0.97-2.73)$ & 0.0651 & $1.70(0.94-3.08)$ & 0.0788 \\
\hline Sex (male vs. female) & $0.43(0.21-0.89)$ & 0.0231 & $0.67(0.28-1.61)$ & 0.3730 \\
\hline PS ( 1 vs. 0$)$ & $2.66(1.31-5.37)$ & 0.0066 & $1.54(0.65-3.69)$ & 0.3284 \\
\hline Vp (Vp1-4 vs. Vp0) & $0.87(0.53-1.42)$ & 0.5777 & & \\
\hline Extrahepatic spread (yes vs. no) & $0.94(0.53-1.66)$ & 0.8213 & & \\
\hline HBV (yes vs. no) & $0.87(0.50-1.52)$ & 0.6276 & & \\
\hline HCV (yes vs. no) & $1.30(0.79-2.13)$ & 0.2981 & & \\
\hline Albumin ( $\geq 3.6$ vs. $<3.6 \mathrm{mg} / \mathrm{dL})$ & $0.55(0.33-0.90)$ & 0.0169 & $0.98(0.48-1.98)$ & 0.9446 \\
\hline Bilirubin ( $\geq 0.8$ vs. $<0.8 \mathrm{mg} / \mathrm{dL}$ ) & $1.22(0.74-2.00)$ & 0.4348 & & \\
\hline ALBI grade (grade 2 vs. grade 1 ) & $1.95(1.11-3.45)$ & 0.0208 & $1.66(0.78-3.53)$ & 0.1855 \\
\hline $\operatorname{AFP}(\geq 400$ vs. $<400 \mathrm{ng} / \mathrm{mL})$ & $2.06(1.24-3.45)$ & 0.0056 & $2.46(1.33-4.55)$ & 0.0043 \\
\hline DCP $(\geq 2,050$ vs $<2,050 \mathrm{mAU} / \mathrm{mL})$ & $1.11(0.67-1.84)$ & 0.6824 & & \\
\hline
\end{tabular}

All covariates were time-fixed except for response, which was time-dependent. OS, overall survival; ALBI grade, albumin-bilirubin grade; AFP, alfa-fetoprotein; DCP, des- $\gamma$-carboxy prothrombin.

\section{Discussion}

This retrospective study analyzed data from the SILIUS study to determine the association between investigator-assessed OR by mRECIST and OS and showed that OR by mRECIST was an independent prognostic factor in patients receiving sorafenib alone, in patients receiving sorafenib plus HAIC, and in the total study population. OS was significantly better in patients with a CR or PR by mRECIST than in those with SD or PD. This study used landmark analyses, which are an established method to eliminate a guarantee-time bias (lead-time bias) $[29,30$, 33]. Responder analysis was performed at three time points $(4,6$, and 8 months); that is, survival at a certain time point was evaluated in relation to the best response by that time point in patients who were still participating in the study at that time. Patients who died before a landmark time point were excluded.

Another established method that eliminates guarantee-time bias (lead-time bias) is to use a multivariable Cox regression model with OR as a time-dependent covariate [29, 30, 33]. This approach adjusts for possible confounding factors, thereby enabling assessment of the effect of tumor response on survival. This statistical method also takes into account timedependent changes in response status.

The important findings of this study are that OR status at 4 months was a strong predictor and prognostic factor for survival in the SOR alone group, and that OR status at 6 months and 8 months was also a significant prognostic factor for OS in both the SOR alone and SOR plus HAIC groups. This implies that survival can be predicted based on OR assessed by mRECIST at a relatively early stage after randomization in systemic therapy.

Multivariable Cox regression analyses in this study also identified PS and AFP levels as independent predictive factors for prognosis in the SILIUS patient population, which is in good agreement with the findings of several previous studies. Landmark analyses and multivariable Cox regression analyses of patients that received sorafenib alone or sorafenib plus HAIC showed similar results, confirming that OR by mRECIST is an independent prognostic factor. The relationship between OR by mRECIST and OS shown in this study is consistent with previously reported results using the database of prospective trials $[26,28,35]$. In one 
Kudo et al.: Objective Response by Systemic Therapy Is an Independent Prognostic Factor for OS

such study, a time-dependent multivariable analysis of data from the phase III BRISK-PS trial clearly showed that OR by mRECIST is an independent predictor of prognosis, and multivariable analyses confirmed that OR is an independent prognostic factor of OS [26]. In a different study, an analysis of pooled data from 2 phase II trials comparing nintedanib to sorafenib revealed a good relationship between OR assessed by mRECIST and OS [28]. OR by mRECIST was also an independent prognostic factor of OS in the REFLECT trial regardless of treatment (lenvatinib or sorafenib) [35].

The present study also confirmed that OR to systemic therapy, sorafenib, assessed by mRECIST is a predictive and prognostic factor for OS [36]. We note that this would not guarantee that the OR is a surrogate endpoint of OS [37].

\section{Conclusions}

This study clearly demonstrates that OR assessed by mRECIST is a predictive and prognostic factor for OS irrespective of the therapy given. These findings also suggest that therapies that result in a high ORR may provide a survival benefit in more patients. Consistent with findings from three previous prospective studies, this study showed that OR by mRECIST in systemic therapy is a predictive and prognostic factor of OS. To provide further evidence of this relationship, more data must be accumulated and ultimately subjected to metaanalysis.

\section{Acknowledgement}

This research was supported by a grant from the Japanese Ministry of Health, Labour and Welfare.

\section{Statement of Ethics}

The study protocol conformed to the ethical guidelines of the World Medical Association Declaration of Helsinki and the protocol was approved by the ethics committees of all participating institutions. Approval code of Kindai University Ethics committee is 22-63. All patients provided written informed consent.

\section{Disclosure Statement}

M.K. has received grants from Taiho Pharmaceuticals, Chugai Pharmaceuticals, Otsuka, Takeda, Sumitomo Dainippon-Sumitomo, Daiichi Sankyo, AbbVie, Astellas Pharma, and Bristol-Myers Squibb; grants and personal fees from MSD, Eisai, and Bayer, and is an adviser for MSD, Eisai, Bayer, Bristol-Myers Squibb, Eli Lilly, and ONO Pharmaceutical. K.U., S.Og., and E.H. have received personal fees and honoraria from Bayer and Eisai. T.O. has received grants from Kowa K.K. and Kyowa Hakko Kirin, grants and personal fees from Novartis, Nippon Boehringer Ingelheim, Dainippon-Sumitomo, Pfizer Jana Inc., Bayer Yakuhin, Chugai Pharmaceuticals, Eli Lilly, Yakuruto Honsha, Ono Pharmceuticals, Eisai, AstraZeneca, Merck Serono, Baxter, Nano Carrier, Zeria Pharmaceuticals, NobelPharma, and TaihoPharmaceuticals, and personal fees from BristolMyers Squibb, Nipponchemofa, EA Pharma, Fujifilm RI Pharma, Nippon Kayaku, Daiichi Sankyo, Celgene, and Teiijin Pharma. T.K. has received personal fees from Gilead, Bristol-Myers Squibb, MSD, and AbbVie. All other authors declare no competing interests.

\section{Funding Sources}

Japanese Ministry of Health, Labour and Welfare. 


\section{Liver Cancer}

Author Contributions

M.K. and K.U. contributed to the study design, data collection, study analysis, manuscript writing, critical review of the manuscript, and final approval of the manuscript submission. Y.C. did the statistical analysis, critical review of the manuscript, and final approval of the manuscript. S.Og., S.Ob., N.I., H.A., H.N., E.H., Y.S., K.H., T.K., K.Y., Y.I., S.I., C.O., T.O., and F.K. contributed to data collection, critical review of the manuscript, and final approval of the manuscript submission.

Y.A. contributed to critical review of the manuscript and final approval of the manuscript submission.

\section{References}

1 Bray F, Ferlay J, Soerjomataram I, Siegel RL, Torre LA, Jemal A. Global cancer statistics 2018: GLOBOCAN estimates of incidence and mortality worldwide for 36 cancers in 185 countries. CA Cancer J Clin. 2018;68(6): 394-424.

2 Galle PR, Forner A, Llovet JM, Mazzaferro V, Piscaglia F, Raoul JL, et al.; EASL Clinical Practice Guidelines. Management of hepatocellular carcinoma. J Hepatol. 2018;69(1):182-236.

3 Kudo M, Izumi N, Sakamoto M, Matsuyama Y, Ichida T, Nakashima 0, et al. Survival Analysis over 28 Years of 173,378 Patients with Hepatocellular Carcinoma in Japan. Liver Cancer. 2016;5(3):190-7.

4 Cabrera R, Nelson DR. Review article: the management of hepatocellular carcinoma. Aliment Pharmacol Ther. 2010;31(4):461-76.

5 Harlan LC, Parsons HM, Wiggins CL, Stevens JL, Patt YZ. Treatment of hepatocellular carcinoma in the community: disparities in standard therapy. Liver Cancer. 2015;4(1):70-83.

6 Kudo M, Finn RS, Qin S, Han KH, Ikeda K, Piscaglia F, et al. Lenvatinib versus sorafenib in first-line treatment of patients with unresectable hepatocellular carcinoma: a randomised phase 3 non-inferiority trial. Lancet. 2018;391(10126):1163-73.

7 Llovet JM, Ricci S, Mazzaferro V, Hilgard P, Gane E, Blanc JF, et al. Sorafenib in advanced hepatocellular carcinoma. N Engl J Med. 2008;359(4):378-90.

8 Cheng AL, Kang YK, Chen Z, Tsao CJ, Qin S, Kim JS, et al. Efficacy and safety of sorafenib in patients in the AsiaPacific region with advanced hepatocellular carcinoma: a phase III randomised, double-blind, placebocontrolled trial. Lancet Oncol. 2009;10(1):25-34.

9 Matsui J, Yamamoto Y, Funahashi Y, Tsuruoka A, Watanabe T, Wakabayashi T, et al. E7080, a novel inhibitor that targets multiple kinases, has potent antitumor activities against stem cell factor producing human small cell lung cancer H146, based on angiogenesis inhibition. Int J Cancer. 2008;122(3):664-71.

10 Matsui J, Funahashi Y, Uenaka T, Watanabe T, Tsuruoka A, Asada M: Multi-kinase inhibitor E7080 suppresses lymph node and lung metastases of human mammary breast tumor MDA-MB-231 via inhibition of vascular endothelial growth factor-receptor (VEGF-R) 2 and VEGF-R3 kinase. Clin Cancer Res. 2008;14:5459-5465.

11 Yamamoto Y, Matsui J, Matsushima T, Obaishi H, Miyazaki K, Nakamura K, et al. Lenvatinib, an angiogenesis inhibitor targeting VEGFR/FGFR, shows broad antitumor activity in human tumor xenograft models associated with microvessel density and pericyte coverage. Vasc Cell. 2014;6(1):18.

12 Tohyama O, Matsui J, Kodama K, Hata-Sugi N, Kimura T, Okamoto K, et al. Antitumor activity of lenvatinib (e7080): an angiogenesis inhibitor that targets multiple receptor tyrosine kinases in preclinical human thyroid cancer models. J Thyroid Res. 2014;2014:638747.

13 Moriguchi M, Aramaki T, Nishiofuku H, Sato R, Asakura K, Yamaguchi K, et al. Sorafenib versus Hepatic Arterial Infusion Chemotherapy as Initial Treatment for Hepatocellular Carcinoma with Advanced Portal Vein Tumor Thrombosis. Liver Cancer. 2017;6(4):275-86.

14 Ueshima K, Kudo M, Takita M, Nagai T, Tatsumi C, Ueda T, et al. Hepatic arterial infusion chemotherapy using low-dose 5-fluorouracil and cisplatin for advanced hepatocellular carcinoma. Oncology. 2010;78 Suppl 1:148-53.

15 Oh MJ, Lee HJ, Lee SH. Efficacy and safety of hepatic arterial infusion chemotherapy for advanced hepatocellular carcinoma as first-line therapy. Clin Mol Hepatol. 2013;19(3):288-99.

16 Woo HY, Bae SH, Park JY, Han KH, Chun HJ, Choi BG, et al. A randomized comparative study of high-dose and low-dose hepatic arterial infusion chemotherapy for intractable, advanced hepatocellular carcinoma. Cancer Chemother Pharmacol. 2010;65(2):373-82.

17 Tsai WL, Lai KH, Liang HL, Hsu PI, Chan HH, Chen WC, et al. Hepatic arterial infusion chemotherapy for patients with huge unresectable hepatocellular carcinoma. PLoS One. 2014;9(5):e92784.

18 Song MJ, Bae SH, Chun HJ, Choi JY, Yoon SK, Park JY, et al. A randomized study of cisplatin and 5-FU hepatic arterial infusion chemotherapy with or without adriamycin for advanced hepatocellular carcinoma. Cancer Chemother Pharmacol. 2015;75(4):739-46.

19 Ma MC, Chen YY, Li SH, Cheng YF, Wang CC, Chiu TJ, et al. Intra-arterial chemotherapy with doxorubicin and cisplatin is effective for advanced hepatocellular cell carcinoma. ScientificWorldJournal. 2014;2014:160138.

20 Obi S, Yoshida H, Toune R, Unuma T, Kanda M, Sato S, et al. Combination therapy of intraarterial 5-fluorouracil and systemic interferon-alpha for advanced hepatocellular carcinoma with portal venous invasion. Cancer. 2006;106(9):1990-7. 
Kudo et al.: Objective Response by Systemic Therapy Is an Independent Prognostic Factor for OS

21 Nouso K, Miyahara K, Uchida D, Kuwaki K, Izumi N, Omata M, et al. Effect of hepatic arterial infusion chemotherapy of 5-fluorouracil and cisplatin for advanced hepatocellular carcinoma in the Nationwide Survey of Primary Liver Cancer in Japan. Br J Cancer. 2013;109(7):1904-7.

22 Ikeda M, Shimizu S, Sato T, Morimoto M, Kojima Y, Inaba Y, et al. Sorafenib plus hepatic arterial infusion chemotherapy with cisplatin versus sorafenib for advanced hepatocellular carcinoma: randomized phase II trial. Ann Oncol. 2016;27(11):2090-6.

23 Ueshima K, Kudo M, Tanaka M, Kumada T, Chung H, Hagiwara S, et al. Phase I/II Study of Sorafenib in Combination with Hepatic Arterial Infusion Chemotherapy Using Low-Dose Cisplatin and 5-Fluorouracil. Liver Cancer. 2015;4(4):263-73.

24 Kudo M, Ueshima K, Yokosuka O, Ogasawara S, Obi S, Izumi N, et al. Sorafenib plus low-dose cisplatin and fluorouracil hepatic arterial infusion chemotherapy versus sorafenib alone in patients with advanced hepatocellular carcinoma (SILIUS): a randomised, open label, phase 3 trial. Lancet Gastroenterol Hepatol. 2018;3(6): 424-32.

25 Lencioni R, Llovet JM. Modified RECIST (mRECIST) assessment for hepatocellular carcinoma. Semin Liver Dis. 2010;30(1):52-60.

26 Lencioni R, Montal R, Torres F, Park JW, Decaens T, Raoul JL, et al. Objective response by mRECIST as a predictor and potential surrogate end-point of overall survival in advanced HCC. J Hepatol. 2017;66(6):116672.

27 Vincenzi B, Di Maio M, Silletta M, D’Onofrio L, Spoto C, Piccirillo MC, et al. Prognostic Relevance of Objective Response According to EASL Criteria and mRECIST Criteria in Hepatocellular Carcinoma Patients Treated with Loco-Regional Therapies: A Literature-Based Meta-Analysis. PLoS One. 2015;10(7):e0133488.

28 Meyer T, Palmer DH, Cheng AL, Hocke J, Loembe AB, Yen CJ. mRECIST to predict survival in advanced hepatocellular carcinoma: Analysis of two randomised phase II trials comparing nintedanib vs sorafenib. Liver Int. 2017;37:1047-1055.

29 Anderson JR, Cain KC, Gelber RD. Analysis of survival by tumor response and other comparisons of time-toevent by outcome variables. J Clin Oncol. 2008;26(24):3913-5.

30 Anderson JR, Cain KC, Gelber RD. Analysis of survival by tumor response. J Clin Oncol. 1983;1(11):710-9.

31 Guidelines for Reporting Clinical Trials. J Clin Oncol. 1986;4(1):1.

32 Simon R, Wittes RE. Methodologic guidelines for reports of clinical trials. Cancer Treat Rep. 1985;69:1-3.

33 Giobbie-Hurder A, Gelber RD, Regan MM. Challenges of guarantee-time bias. J Clin Oncol. 2013;31(23):2963-9.

34 Simon R, Makuch RW. A non-parametric graphical representation of the relationship between survival and the occurrence of an event: application to responder versus non-responder bias. Stat Med. 1984;3(1):35-44.

35 Kudo M, Finn RS, Qin S, Han KH, Ikeda K, Cheng AL, et al. Analysis of survival and objective responses in patients with hepatocellular carcinoma in a phase 3 study of lenvatinib (REFLECT) [abstract]. J Clin Oncol 37, 2019;37(suppl):186.

36 Ballman KV. Biomarker: predictive or Prognostic? J Clin Oncol. 2015;33(33):3968-71.

37 Baker SG, Kramer BS. A perfect correlate does not a surrogate make. BMC Med Res Methodol. 2003;3(1):16. 